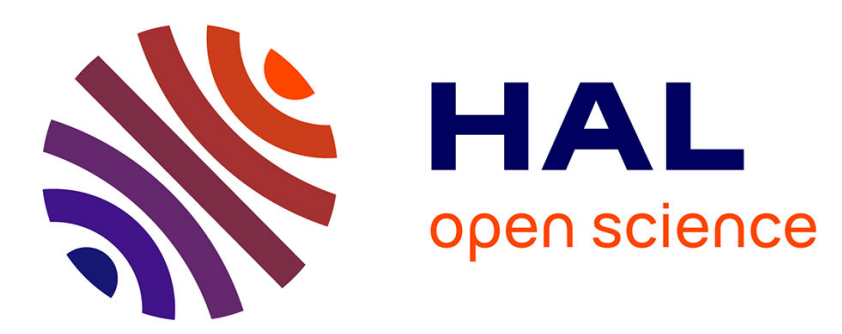

\title{
Adaptive feedback noise attenuation in the presence of plant uncertainties -A Dual Youla-Kucera approach
}

Ioan Doré Landau, Bernard Vau, Gabriel Buche

\section{To cite this version:}

Ioan Doré Landau, Bernard Vau, Gabriel Buche. Adaptive feedback noise attenuation in the presence of plant uncertainties -A Dual Youla-Kucera approach. ECC 2021 - 20th European Control Conference, European Control Association, Jun 2021, Rotterdam (virtual), Netherlands. 10.23919/ECC54610.2021.9655106 . hal-03196442

\section{HAL Id: hal-03196442 \\ https://hal.science/hal-03196442}

Submitted on 12 Apr 2021

HAL is a multi-disciplinary open access archive for the deposit and dissemination of scientific research documents, whether they are published or not. The documents may come from teaching and research institutions in France or abroad, or from public or private research centers.
L'archive ouverte pluridisciplinaire HAL, est destinée au dépôt et à la diffusion de documents scientifiques de niveau recherche, publiés ou non, émanant des établissements d'enseignement et de recherche français ou étrangers, des laboratoires publics ou privés. 


\title{
Adaptive feedback noise attenuation in the presence of plant uncertainties - A Dual Youla-Kucera approach
}

\author{
Ioan Dore Landau ${ }^{1}$ and Bernard Vau ${ }^{2}$ and Gabriel Buche ${ }^{1}$
}

\begin{abstract}
This paper presents an adaptive algorithm for attenuation of tonal and narrow band noise in the case of large uncertainties of the compensatory path. This scheme implements the Internal Model Principle (IMP) for canceling disturbances, combined with the Youla Kucera (YK) parametrization which allows to directly tune the disturbance compensation filter. Plant uncertainties are represented by means of the DualYoula-Kucera parameterization, and an overparametrization of the adaptive disturbance compensation filter is proposed to cope with the uncertain plant. In addition, a frequency condition has to be satisfied by an appropriate design of the central controller. The experimental validation of the design is done on a relevant active noise control test bench.
\end{abstract}

\section{INTRODUCTION}

The issue of rejecting multiple narrow band disturbances with unknown and time-varying characteristics can be addressed efficiently by an adaptive feedback control solution. The principle of adaptive feedback is to use the Internal Model Principle (IMP) of Wohnam [1], combined with a Youla-Kucera parametrization of the controller, allowing to adaptively tune its parameters. The use of this approach in active noise attenuation is presented in [2], [3], [4] and this method has been widely employed in active vibration control [5], [6]. In these references, it is assumed that the plant model is known and almost constant. However, in several situations, large variations of the plant model can occur, leading potentially to closed-loop instability. In order to represent the plant uncertainties, a dual Youla-Kucera parameterization can be used [7], [8]. In [9] it is argued that overparameterizing the Youla-Kucera filter of the adaptive controller one can enhance its robustness with respect to plant uncertainty, and this idea has been exploited in [8], where it is shown that the overparameterization should do simultaneously two tasks: 1) verify the internal model principle 2) guaranteeing closed-loop stability. Even assuming that one knows the plant uncertainty, it results that two frequency conditions for stability appear. The first condition depends upon the discrepancies between the nominal and the real model and can be influenced by the design of the central stabilizing controller. The second condition requires a specific design of the central controller. Furthermore, because of overparametrization, the argument of richness excitation is not sufficient to ensure the estimated parameters convergence. For this reason the Parameter Adaptation Algorithm (PAA) introduced in [8] is completed with a projection procedure to maintain the

\footnotetext{
${ }^{1}$ Gipsa-lab, 38000 Grenoble, France email: ioan-dore.landau@gipsalab.grenoble-inp.fr

${ }^{2}$ Ixblue, 12 avenue des coquelicots, 94385 Bonneuil-Sur-Marne, France email: bernard-vau@ixblue.com
}

parameters within a certain domain of variation.

Many of these aspects have been discussed in [10]. However in [10] the design procedure has been illustrated for the case of asymptotically stable plants and without using a central stabilizing controller. While this choice simplifies the design, the domain of operation is limited by the discrepancy between the design model and the real model. In the present paper one considers the use of a central stabilizing controller and this allows to expand the region of operation up to the region of operation covered in the absence of uncertainties on the model of the secondary path. The paper is organized as follows: In Section II the experimental setup will be described. In Section III the basic equations of the algorithm are presented, and the conditions for closed-loop stability are given, which leads to propose a methodology for the design of the central controller in Section IV. Experimental results obtained on an active noise control test-bench are presented in Section V.

\section{EXPERIMENTAL SETUP}

The test bench allows to test active noise control in pipes for various physical configurations. The detailed scheme of test bench with the control loop is shown in Fig. 1 and the views of the two implementations which will be considered subsequently are shown in Fig. 2.

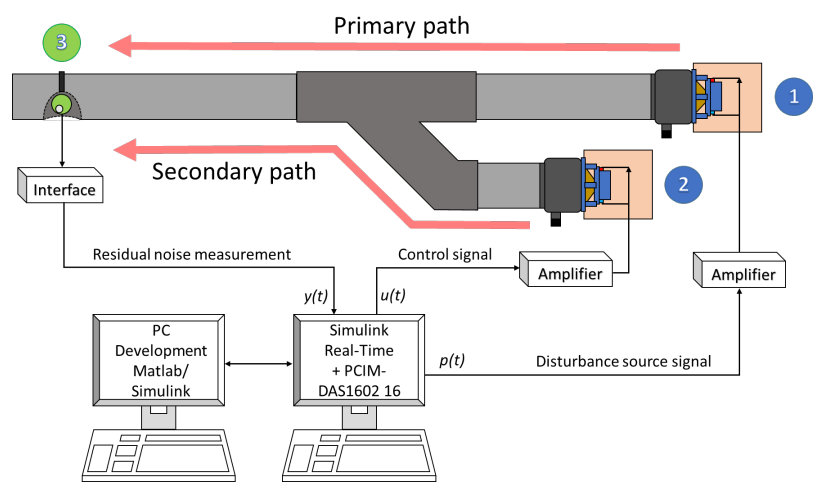

Fig. 1: Duct active noise control test bench diagram.

In Fig. 1, the speaker used as the source of disturbances is labelled as 1, while the control speaker is marked as 2. At pipe's open end, the microphone that measures the system's output (residual noise ) is denoted as 3. The control signal is denoted $u(t)$, the residual noise is denoted $y(t)$. The transfer function between the disturbance's speaker and the microphone $(1 \rightarrow 3)$ is called Primary Path, while 


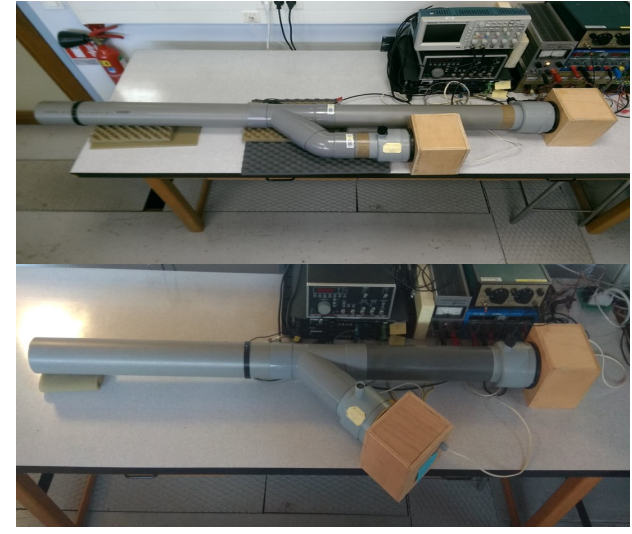

Fig. 2: Duct active noise control test bench - Configuration Go (top), configuration $\mathrm{G}$ (bottom)

the transfer function between the control speaker and the microphone $(2 \rightarrow 3)$ is denoted Secondary Path. These marked paths have a double differentiator behaviour, since as input we have the voice coil displacement and as output the air acoustic pressure. Both speakers are connected to a PC target computer with Simulink Real-time ${ }^{\circledR}$ environment through a pair of high definition power amplifiers and a data acquisition board. A second computer is used for development, design and operation with Matlab ${ }^{\circledR}$. The sampling frequency is $f_{s}=2500 \mathrm{~Hz}$.

\section{SYSTEM STRUCTURE AND CONTROLLER DESIGN}

The nominal plant model used for controller design (design model $)^{1}$ is denoted $G_{o}\left(q^{-1}\right)$ and is described by:

$$
G_{o}\left(q^{-1}\right)=\frac{B_{o}\left(q^{-1}\right)}{A_{o}\left(q^{-1}\right)}
$$

with:

$$
\begin{array}{r}
A_{o}\left(q^{-1}\right)=1+a_{1} q^{-1}+\cdots+a_{n_{A}} q^{-n_{A}} ; \\
B_{o}\left(q^{-1}\right)=b_{1} q^{-1}+\cdots+b_{n_{B}} q^{-n_{B}}=q^{-1} B_{o}^{*}\left(q^{-1}\right) ; \\
B_{o}^{*}\left(q^{-1}\right)=b_{1}+\cdots+b_{n_{B}} q^{-n_{B}+1} ;
\end{array}
$$

where $A\left(q^{-1}\right), B\left(q^{-1}\right), B^{*}\left(q^{-1}\right)$ are polynomials in the delay operator $q^{-1}$ and $n_{A_{o}}, n_{B_{o}}$ and $n_{B}-1$ represent their orders. The real plant model denoted $G\left(q^{-1}\right)$ has the same structure as $G_{o}\left(q^{-1}\right)$,

$$
G\left(q^{-1}\right)=\frac{B\left(q^{-1}\right)}{A\left(q^{-1}\right)}
$$

but the order of the various polynomials $\left(n_{A}, n_{B}\right)$, the delay $d$ and the parameters are unknown.

The control structure includes a central controller $C_{o}$

$$
C_{o}\left(q^{-1}\right)=\frac{R_{o}\left(q^{-1}\right)}{S_{o}\left(q^{-1}\right)}=\frac{R_{o}^{\prime}\left(q^{-1}\right) H_{R}\left(q^{-1}\right)}{S_{o}^{\prime}\left(q^{-1}\right) H_{S}\left(q^{-1}\right)}
$$

and $H_{r}\left(q^{-1}\right)$ and $H_{S}\left(q^{-1}\right)$ are some fixed parts which may be imposed during the controller synthesis (See [11]). This

\footnotetext{
${ }^{1}$ In active vibration and noise control the plant is called "secondary path" or "compensation path"
}

controller stabilizes the nominal model, meaning that the closed-loop polynomial

$$
P_{o}\left(q^{-1}\right)=A_{o}\left(q^{-1}\right) S_{o}\left(q^{-1}\right)+B_{o}\left(q^{-1}\right) R_{o}\left(q^{-1}\right)
$$

has (by design) all its roots strictly inside the unit circle. The following hypothesis is made throughout the paper:

H1- The real plant model $G$ is also stabilized by the central controller $C_{o}$.

One assumes that the output disturbance $d(t)$ results from the filtering of a Dirac impulse $\delta(t)$

$$
d(t)=\frac{N_{d}\left(q^{-1}\right)}{D_{d}\left(q^{-1}\right)} \delta(t)
$$

where $D_{d}\left(q^{-1}\right)$ has all its roots on the unit circle.

One considers a Youla-Kucera (YK) parametrization of the controller [12]. For the purpose of this paper the YoulaKucera filter $Q\left(q^{-1}\right)$ is considered to be a polynomial of the form:

$$
Q\left(q^{-1}\right)=q_{o}^{Q}+q_{1}^{Q} q^{-1}+\ldots+q_{n_{Q}}^{Q} q^{-n_{Q}} .
$$

which is fed by the signal $w(t)$

$$
w(t)=-B_{o}\left(q^{-1}\right) u(t)+A_{o}\left(q^{-1}\right) y(t)
$$

Note that for the nominal case

$$
w(t)=A_{o}\left(q^{-1}\right) d(t)
$$

which can be interpreted as a stable observation of the disturbance [12]. In the presence of the Youla-Kucera parametrization, the resulting controller becomes:

$$
u(t)=-C\left(q^{-1}\right) y(t)=-\frac{R\left(q^{-1}\right)}{S\left(q^{-1}\right)} y(t)
$$

where

$$
\begin{array}{r}
R\left(q^{-1}\right)=R_{o}+A_{o} H_{R} H_{S} Q \\
S\left(q^{-1}\right)=S_{o}-B_{o} H_{R} H_{S} Q
\end{array}
$$

To compute $Q\left(q^{-1}\right)$ in order that the controller contains the internal model of the disturbance (required by the Internal Model Principle for asymptotic rejection of the disturbance), one has to solve $\left(S=S^{\prime} D_{d}\right)$ :

$$
S^{\prime} D_{d}+H_{R} H_{S} B_{o} Q=S_{o}
$$

where $S_{o}$ and $Q$ are the unknown terms.

Fig. 3 gives the block diagram of the true control system where in addition the uncertainty on the plant model is taken into account using the dual Youla-Kucera parametrization of the plant model. The dual Youla-Kucera filter is defined as

$$
\frac{\Delta\left(q^{-1}\right)}{\Gamma\left(q^{-1}\right)}
$$

The polynomial $\Gamma\left(q^{-1}\right)$ is monic with all its roots strictly inside the unit circle, and the polynomial $\Delta\left(q^{-1}\right)$ has no direct transmission. Under these assumptions, the uncertain plant $G\left(q^{-1}\right)$ can be written

$$
G\left(q^{-1}\right)=\frac{\Gamma\left(q^{-1}\right) B_{o}\left(q^{-1}\right)+\Delta\left(q^{-1}\right) S_{o}\left(q^{-1}\right)}{\Gamma\left(q^{-1}\right) A_{o}\left(q^{-1}\right)-\Delta\left(q^{-1}\right) R_{o}\left(q^{-1}\right)}
$$




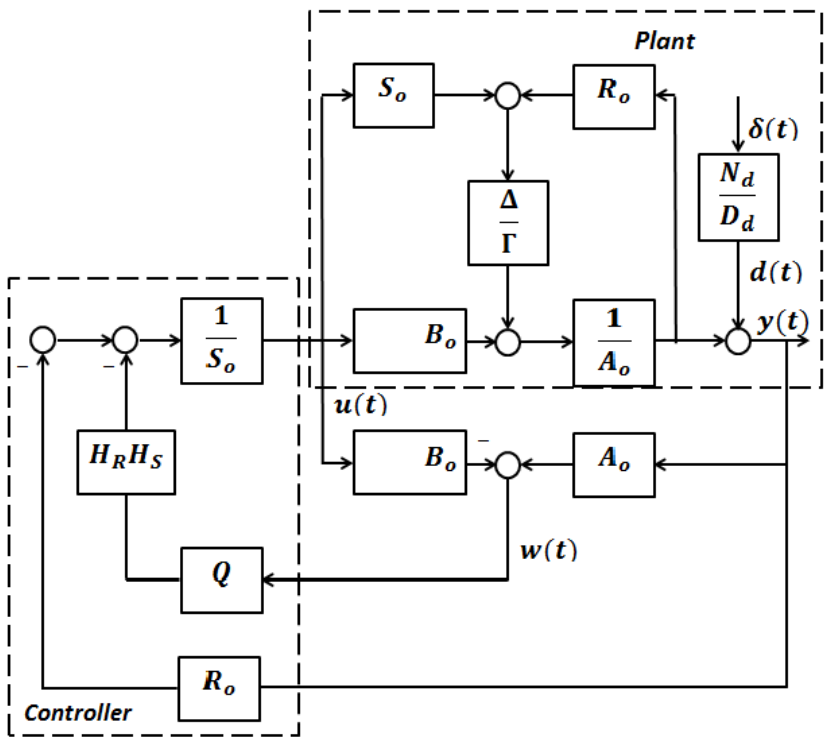

Fig. 3: Global block diagram of the uncertain system and the controller including the Q-filter

In the presence of uncertainties in the plant model, the following relation holds $(d(t)$ is the disturbance):

$$
y(t)=\frac{\Gamma A_{o}-\Delta R_{o}}{P_{o}} \frac{S_{o}-Q H_{R} H_{S} B_{o}}{\Gamma+Q H_{R} H_{S} \Delta} d(t)
$$

One can notice that for $G\left(q^{-1}\right) \neq G_{o}\left(q^{-1}\right)$, a stability condition appears :

$$
\Gamma\left(q^{-1}\right)+Q\left(q^{-1}\right) H_{R}\left(q^{-1}\right) H_{S}\left(q^{-1}\right) \Delta\left(q^{-1}\right)
$$

must have all its roots strictly inside the unit circle. In this context, one has the following result for $w(t)$ :

Lemma 1: In case of model uncertainty expressed with the Dual Youla-Kucera parameterization as in (14), one has

$$
w(t)=\frac{\Gamma\left(q^{-1}\right) A o\left(q^{-1}\right)-\Delta\left(q^{-1}\right) R_{o}\left(q^{-1}\right)}{\Gamma\left(q^{-1}\right)+Q\left(q^{-1}\right) H_{R}\left(q^{-1} H_{S}\left(q^{-1}\right) \Delta\left(q^{-1}\right)\right.} d(t)
$$

\section{Proof: See [10] $\square$}

As a consequence, in order that $w(t)$ be an observation of $d(t)$ (which is bounded), the polynomial (16) must have all its roots strictly inside the unit circle. This can be summarized as follows:

Lemma 2: For the case of plant uncertainty represented by the dual Youla Kucera parametrization and in the presence of a bounded disturbance $d(t)$, the signals $y(t), u(t)$ and $w(t)$ will be bounded provided that the polynomial (16) will have all its roots strictly inside the unit circle.

Since $G\left(q^{-1}\right)$ differs from $G_{o}\left(q^{-1}\right)$, the minimal order solution for $Q$ (defined in (7)) satisfying the internal model principle, does not necessarily leads to the stability of the closed-loop. In such a situation, one can augment the order of $Q\left(q^{-1}\right)$. One has the following result about the existence of a polynomial $Q\left(q^{-1}\right)$ with a degree $n_{Q}<\infty$ guaranteeing closed-loop stability and satisfying the internal model principle:
Lemma 3: The existence of a finite dimensional $Q$ which stabilizes the closed-loop and asymptotically rejects the disturbance $d(t)$ is assured (sufficient condition) if:

- For the frequencies $\omega_{j}$ of the disturbance corresponding to $D_{d}\left(e^{-i \omega_{j}}\right)=0$, the following inequality is satisfied:

$$
\begin{aligned}
\left|\frac{S_{o}\left(e^{-i \omega_{j}}\right)}{B_{o}\left(e^{-i \omega_{j}}\right)}\right| & < \\
& \left|\frac{A\left(e^{-i \omega_{j}}\right) S_{o}\left(e^{-i \omega_{j}}\right)+B\left(e^{-i \omega_{j}}\right) R_{o}\left(e^{-i \omega_{j}}\right)}{B\left(e^{-i \omega_{j}}\right) A_{o}\left(e^{-i \omega_{j}}\right)-A\left(e^{-i \omega_{j}}\right) B_{o}\left(e^{-i \omega_{j}}\right)}\right|
\end{aligned}
$$

- For all other frequencies, the following inequality is satisfied:

$$
\begin{aligned}
& \left|Q\left(e^{-i \omega}\right)\right|< \\
& \left|\frac{A\left(e^{-i \omega}\right) S_{o}\left(e^{-i \omega}\right)+B\left(e^{-i \omega}\right) R_{o}\left(e^{-i \omega}\right)}{B\left(e^{-i \omega}\right) A_{o}\left(e^{-i \omega}\right)-A\left(e^{-i \omega}\right) B_{o}\left(e^{-i \omega}\right)}\right| \cdots \\
& \cdots \times\left|\frac{1}{H_{R}\left(e^{-i \omega}\right) H_{S}\left(e^{-i \omega}\right)}\right|
\end{aligned}
$$

Proof: See [10].

\section{Adaptive disturbance rejection}

In the presence of unknown narrow band disturbances the polynomial $D_{d}\left(q^{-1}\right)$ is unknown. In this situation one can consider a Q-filter with adjustable parameters:

$$
\hat{Q}\left(q^{-1}, t\right)=\hat{q}_{o}^{Q}(t)+\cdots \hat{q}_{n Q}^{Q}(t) q^{-n Q}
$$

and the objective is to find a parameter adaptation algorithm driving this parameters towards the values assuring asymptotic rejection of the disturbance. We will follow up to certain extent the development procedure described in [12], however including from the beginning the presence of model uncertainties described by the dual Youla Kucera representation and the use of a $\mathrm{Q}$ filter of higher order that the minimal one used for the nominal case (when $G=G_{o}$ ). When using a Q-filter with constant coefficients the output of the system $y(t)$ in the presence of a disturbance $d(t)$ can be expressed as:

$$
y(t)=\frac{\Gamma A_{o}-\Delta R_{o}}{P_{o}} \frac{\left(S_{o}-B_{o} \hat{Q} H_{R} H_{S}\right)}{\Gamma+\hat{Q} H_{R} H_{S} \Delta} d(t)
$$

By combining (21) and (17), one obtains

$$
y(t)=\left(\frac{S_{o}}{P_{o}}-\hat{Q} \frac{H_{R} H_{S} B_{o}}{P_{o}}\right) w(t)
$$

Since the objective is to drive $y(t)$ asymptotically to zero, it is logical to consider this variable as an adaptation error which will be denoted $\varepsilon(t)$. Now replacing in (22) the fixed value of $Q$ by an estimation of $\hat{Q}$ one gets an expression for the a-priori adaptation error $\varepsilon^{o}(t+1)$ :

$$
\varepsilon^{o}(t+1)=\left(\frac{S_{o}}{P_{o}}-\hat{Q}\left(q^{-1}, t\right) \frac{H_{R} H_{S} B_{o}}{P_{o}}\right) w(t+1)
$$

One can define the a-posteriori adaptation as:

$$
\varepsilon(t+1)=\left(\frac{S_{o}}{P_{o}}-\hat{Q}\left(q^{-1}, t+1\right) \frac{H_{R} H_{S} B_{o}}{P_{o}}\right) w(t+1)
$$


Taking into account Eq.(12) (resulting from the application of the IMP principle for the case of known disturbances) Eq.(24) can rewritten as:

$$
\begin{array}{r}
\varepsilon(t+1)=\left(Q\left(q^{-1}\right)-\hat{Q}\left(q^{-1}, t+1\right)\right) \frac{B_{o}^{*} H_{R} H_{S}}{P_{o}} w(t) \\
+v(t+1)
\end{array}
$$

where $v(t+1)=\frac{D_{p}\left(q^{-1}\right) S^{\prime}\left(q^{-1}\right)}{P_{o}\left(q^{-1}\right)} w(t+1)$ is a signal which tends towards 0 . Set

$$
w_{2}(t)=\frac{B_{o}^{*}}{P_{o}} w(t)
$$

The a-posteriori adaptation error can be expressed under the form [13]:

$$
\varepsilon(t+1)=H\left(q^{-1}\right)(\theta-\hat{\theta}(t+1))^{T} \phi(t)
$$

where

$$
\begin{gathered}
\phi^{T}(t)=\left[w_{2}(t) \cdots w_{2}\left(t-n_{Q}\right)\right] \\
\theta^{T}=\left[q_{o}^{Q} \cdots q_{n_{Q}}^{Q}\right] \\
\hat{\theta}^{T}(t)=\left[\hat{q}_{o}^{Q}(t) \cdots \hat{q}_{n_{Q}}^{Q}(t)\right]
\end{gathered}
$$

and here, owing to (27)

$$
H\left(q^{-1}\right)=1
$$

Taking into account the fact that the order of polynomial $\hat{Q}$ is higher than the minimal order required by the IMP, the parameter adaptation algorithm proposed in [12] and [8] has to be completed with a projection of the estimated parameter vector on a bounded domain in order to prove the stability of the adaptive control scheme (see [13] p.340). The adaptation algorithm to be used is

$$
\begin{gathered}
\hat{\theta}(t+1)=\hat{\theta}_{p}(t)+F(t) \phi(t) \varepsilon(t+1) \\
\varepsilon(t+1)=\frac{\varepsilon^{o}(t+1)}{1+\phi^{T} F(t) \phi(t)} \\
F(t+1)^{-1}=\lambda_{1} F(t)^{-1}+\lambda_{2} \phi(t) \phi^{T}(t) \\
0<\lambda_{1}<1 \quad 0 \leq \lambda_{2}<2, F_{0}>0
\end{gathered}
$$

where $\hat{\theta}_{p}(t)$ is the projection of $\hat{\theta}(t)$ which is computed as follows:

$$
\begin{aligned}
& \hat{\theta}^{\prime}(t)=F(t)^{-1 / 2} \hat{\theta}(t) \\
& \hat{\theta}_{p}^{\prime}(t)=\hat{\theta}^{\prime}(t) \quad \text { if } \theta^{\prime}(t+1) \in \mathscr{D}^{\prime} \\
& \hat{\theta}_{p}^{\prime}(t)=\perp \text { proj of } \theta^{\prime}(t) \text { on } \mathscr{D}^{\prime} \quad \text { if } \theta^{\prime}(t) \notin \mathscr{D}^{\prime} \\
& \hat{\theta}_{p}(t)=F(t)^{1 / 2} \hat{\theta}_{p}^{\prime}(t)
\end{aligned}
$$

The projection domain $\mathscr{D}^{\prime}$ is defined as follows:

$$
\hat{\theta} \in \mathscr{D}, \quad \hat{\theta}^{\prime}(t)=F(t)^{-1 / 2} \hat{\theta}(t) \in \mathscr{D}^{\prime}
$$

where the projection domain $\mathscr{D}$ is such that:

$$
\mathscr{D}: \quad\|\hat{\boldsymbol{\theta}}(t)\|_{2}^{2}<\mathscr{R}<\infty
$$

Two particular choices for the adaptation gain are used mainly in practice in order to assure the alertness of the adaptation with respect to possible variations of the disturbance characteristics ${ }^{2}$ :

- Constant trace- for a constant ratio $\lambda_{1}(t) / \lambda_{2}(t), \lambda_{1}(t)$ is chosen such that the trace of the adaptation gain matrix $F(t)$ remain constant $\left(\operatorname{trace} F(t)=\operatorname{trace} F_{0}\right)$

- Constant gain $\lambda_{1}(t)=1, \lambda_{2}(t)=0$ and therefore $F(t)=$ $F_{0}$ (usually $F_{0}=\alpha I$ )

When using a constant adaptation gain, the change of coordinates introduced in Eqs. (34) is no more necessary (see [13], pp. 340-343).

The stability analysis of this adaptive control algorithm can be found in[10] and is omitted.

\section{DESIGN OF THE CENTRAL CONTROLLER}

The central controller design has several objectives: 1) Stabilize the nominal model and the uncertain model. 2) Try to maximize the frequency domain where condition (18) is satisfied. 3) Avoid low values of the right hand side of inequality (19). The central controller polynomials $S_{o}$ and $R_{o}$ are determined by :

a) Selecting the fixed parts $H_{S}, H_{R}$, in order to satisfy conditions (18) and (19). For this purpose, the zeros of $H_{S}$ and $H_{R}$ are low damped complex ones and their frequencies are spread over all spectrum. A pair of closed-loop poles (for the nominal system) is assigned to each pair of complex zeros of $H_{S}$ or $H_{R}$ such that the corresponding complex poles have the same frequency but with a higher damping. In order to achieve this objective, the transfer function operators $\frac{H_{S}\left(q^{-1}\right)}{P_{S}\left(q^{-1}\right)}$ and $\frac{H_{R}\left(q^{-1}\right)}{P_{R}\left(q^{-1}\right)}$ are chosen as the discretization of series of continuous resonating filters, each filter being such that $\frac{\frac{s^{2}}{\omega^{2}}+2 \frac{\zeta_{N} s}{\omega}+1}{\frac{s^{2}}{\omega^{2}}+2 \frac{\zeta_{D}}{\omega}+1}$. Their damping factors are chosen as to satisfy $\stackrel{\omega^{2}}{o} \leq \zeta_{N}<1, o<\zeta_{D} \leq 1$, and $\zeta_{N}<\zeta_{D}$.

b) Forcing some closed-loop poles to be the poles of the nominal systems (the roots of $A_{o}$ ). This corresponds to an internal model strategy, which is recognized to have a good robustness with respect to uncertainties.

Finally $S_{o}=S^{\prime} H_{S}$ and $R_{o}=R^{\prime} H_{R}$ are computed by solving the Bézout equation

$$
A_{o} H_{S} S^{\prime}+B_{o} H_{R} R^{\prime}=A_{o} P_{R} P_{S}
$$

Figure 4 compares the modules of $\frac{S_{o}}{B_{o}}$ and $\frac{A S_{o}+B R_{o}}{B A_{o}-A B_{o}}$, and shows that condition (18) is satisfied up to $270 \mathrm{~Hz}$, by using the designed central controller. In [10] the central controller has been suppressed and the highest frequency for which condition (18) is satisfied, is around $230 \mathrm{~Hz}$.

\section{EXPERIMENTAL RESULTS}

The objective of the experimental validation is to assess the performance of the overparametrized YK adaptive feedback scheme on the configuration $G$ using the model of the configuration $G_{o}$ for implementing the YK observer and using the central controller designed in Section IV. Several type of disturbances will be considered.

\footnotetext{
${ }^{2}$ For other options see [12], [13]
} 


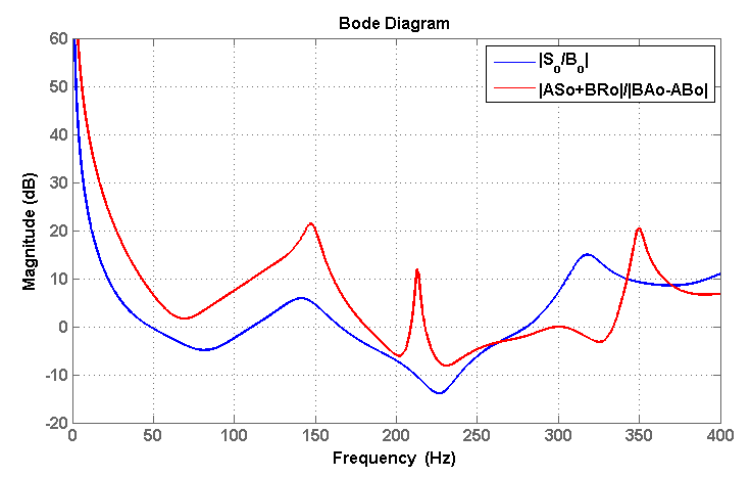

Fig. 4: Comparison of $\left|\frac{S_{o}}{B_{o}}\right|$ and $\left|\frac{A S_{o}+B R_{o}}{B A_{o}-A B_{o}}\right|$

\section{A. System identification}

The secondary path models of the two configurations have been identified from experimental data using the methodology described in [14]. Fig. 5 shows the frequency characteristics of the identified models. There are important differences between the two models. These characteristics present multiple resonances (low damped complex poles) ${ }^{3}$ and anti-resonances (low damped complex zeros). The orders of the two models are summarized in Table I.

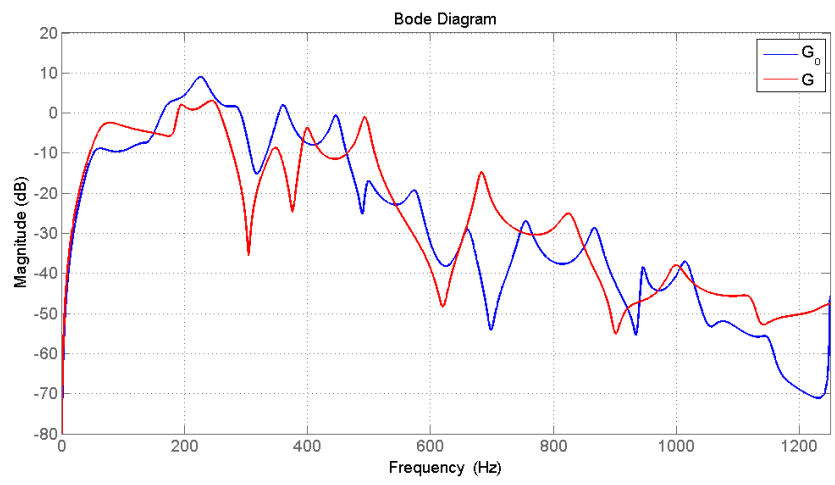

Fig. 5: Bode diagrams of the nominal and uncertain plants

\begin{tabular}{c|c|c|c}
\hline Model & $n_{A}$ & $n_{B}$ & $d$ \\
\hline \hline Secondary path Go & 38 & 32 & 8 \\
\hline Secondary path G & 27 & 20 & 7 \\
\hline
\end{tabular}

TABLE I: Orders of the identified models.

\section{B. Single sinusoidal disturbance}

Fig. 6 shows the response of the residual noise for a sequence of step changes in the frequency of the disturbance around $170 \mathrm{~Hz}$. The system operates in open loop for $5 \mathrm{~s}$ and then at $\mathrm{t}=25 \mathrm{~s}$ a step of $-10 \mathrm{~Hz}(160 \mathrm{~Hz})$ is applied. Then the system returns to the nominal frequency at $t=50$ $\mathrm{s}$ and at $\mathrm{t}=75 \mathrm{~s}$ a step of $+10 \mathrm{~Hz}(180 \mathrm{~Hz})$ is applied. The corresponding evolution of the parameters is shown in Fig. 7. The Q filter has in this case 50 parameters $(n Q=49)$

\footnotetext{
${ }^{3}$ The lowest damping is around 0.01 .
}

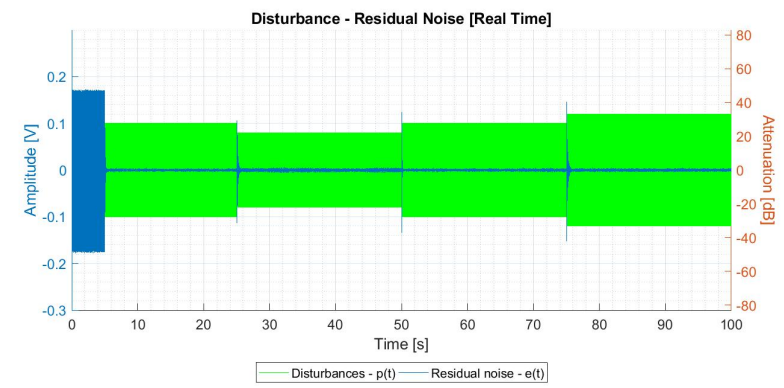

Fig. 6: Time response of the residual noise for step changes in the frequency of the disturbance around $170 \mathrm{~Hz}(50$ par.)

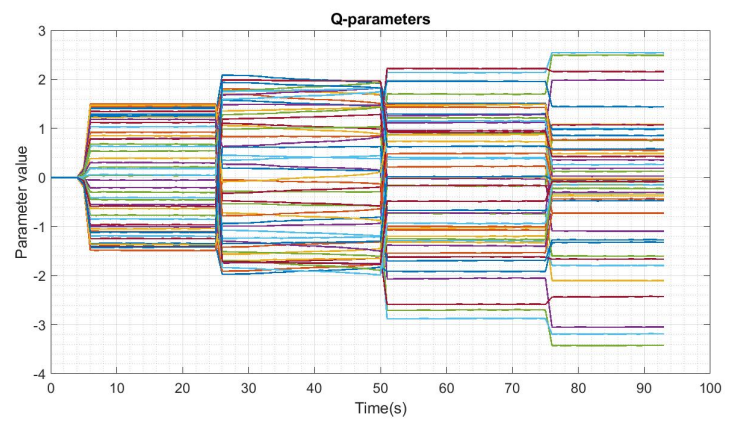

Fig. 7: Evolution of the parameters for step changes in the frequency of the disturbance around $160 \mathrm{~Hz}$ (50 par.)

One observes an excellent attenuation of the disturbance and a very fast adaptation transient. The attenuation ${ }^{4}$ achieved are: $78.43 \mathrm{~dB}$ (5 to $25 \mathrm{~s}$ ) and $77.51 \mathrm{~dB}$ (50 to 75 s) for $170 \mathrm{~Hz}, 72.82 \mathrm{~dB}$ for $160 \mathrm{~Hz}$ and $77.67 \mathrm{~dB}$ for 180 $\mathrm{Hz}$.

\section{Adaptive attenuation of interferences}

Fig. 8 shows the capability of the control scheme to strongly attenuate interference (interference occurs when two sinusoidal disturbances have very close frequencies). A couple of sinusoids at $150 \mathrm{~Hz}$ and $150.5 \mathrm{~Hz}$ is applied. Then at $\mathrm{t}=25 \mathrm{~s}$ one switches to $140 \mathrm{~Hz}$ and $140.3 \mathrm{~Hz}$, at $\mathrm{t}=50$ $\mathrm{s}$ one returns to $150 \mathrm{~Hz}$ and $150.5 \mathrm{~Hz}$ and at $\mathrm{t}=75 \mathrm{~s}$ one switches to $160 \mathrm{~Hz}$ and $160.2 \mathrm{~Hz}$. The system operates in open loop for the first $5 \mathrm{~s}$. The corresponding evolution of the parameters (50 parameters) is shown in Fig. 9.

\section{Two sinusoidal disturbances of distinct frequencies}

To asymptotically reject the effect of two simultaneous sinusoidal disturbances with distinct frequencies and to assure simultaneously the system stability, the dimension of the $Q$ filter has been augmented to 60 parameters $(n Q=59)$. Fig. 10 displays the time response of the residual error for the rejection of two sinusoidal disturbances located at 80 $\mathrm{Hz}$ and $180 \mathrm{~Hz}$ (open loop operation for the first $5 \mathrm{~s}$ ). An attenuation of $86.23 \mathrm{~dB}$ is achieved. Fig. 11 shows the power spectral density in open and in closed loop.

\footnotetext{
${ }^{4}$ Attenuation is defined as the ratio between the variance of the residual noise in open loop and the variance of the residual noise in closed loop
} 


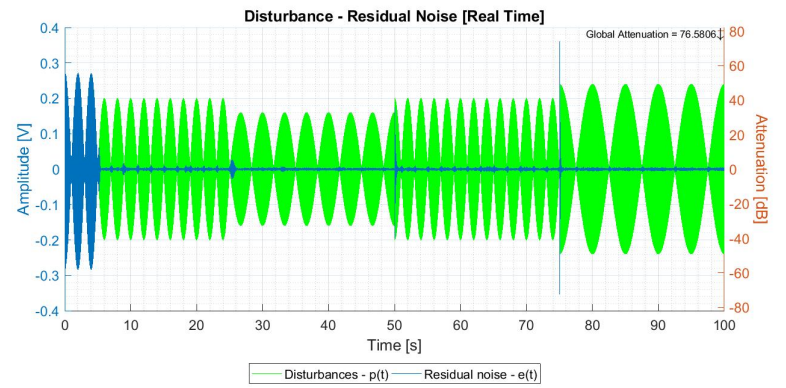

Fig. 8: Time response of the residual noise for step changes in the frequencies of an interference phenomenon (50 par.)

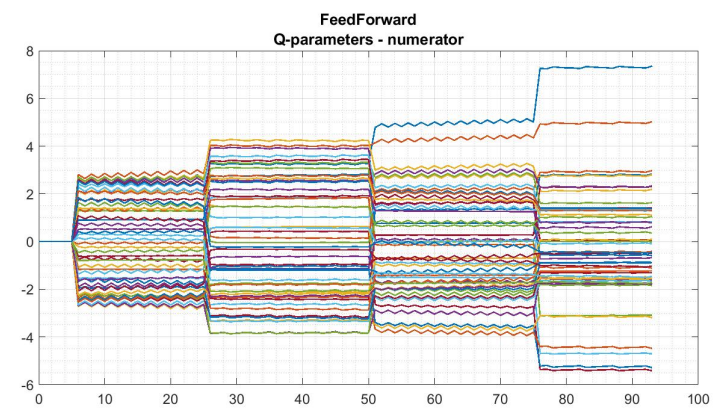

Fig. 9: Evolution of the parameters for step changes in the frequencies of an interference phenomenon (50 par.)

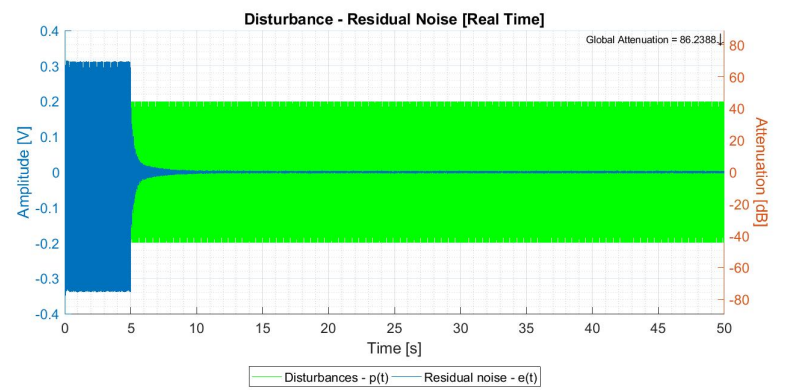

Fig. 10: Evolution of the residual noise for two sinusoïdal disturbances $(80 / 180 \mathrm{~Hz}-60$ parameters).

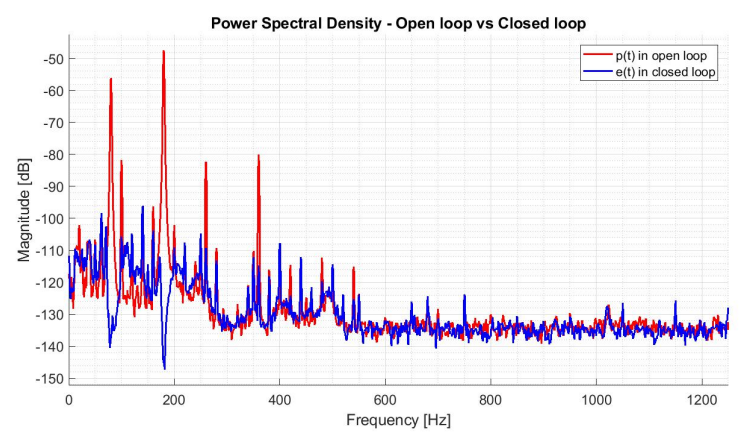

Fig. 11: Power Spectral density of the residual noise in open loop and in closed loop for three sinusoidal disturbances (80/180 Hz - 60 parameters).

\section{CONCLUSION}

The paper has shown that large model uncertainties in the model of the compensatory path used in adaptive feedback attenuation of noise can be handled by overparametrization of the Youla Kucera Q filter. Further experimental results will be provided for the final version of the paper.

\section{REFERENCES}

[1] B. Francis, W. Wonham, The internal model principle of control theory, Automatica 12 (5) (1976) 457 - 465.

[2] F. Ben Amara, P. Kabamba, A. Ulsoy, Adaptive sinusoidal disturbance rejection in linear discrete-time systems - Part I: Theory, Journal of Dynamic Systems Measurement and Control 121 (1999) 648-654.

[3] F. Ben Amara, P. Kabamba, A. Ulsoy, Adaptive sinusoidal disturbance rejection in linear discrete-time systems - Part II: Experiments, Journal of Dynamic Systems Measurement and Control 121 (1999) 655-659.

[4] I. Landau, R. Melendez, L.Dugard, G. Buche, Robust and adaptive noise attenuation in ducts, IEEE Trans. on Control Systems Technology 37 (2) (2019) 872-879.

[5] I. D. Landau, A. C. Silva, T.-B. Airimitoaie, G. Buche, M. Noé, Benchmark on adaptive regulation - rejection of unknown/time-varying multiple narrow band disturbances, European Journal of Control 19 (4) (2013) $237-252$.

[6] I. D. Landau, T.-B. Airimitoaie, A. Castellanos Silva, A. Constantinescu, Adaptive and Robust Active Vibration Control-Methodology and Tests, Advances in Industrial Control, Springer, London, 2017.

[7] C. Kinney, H. Fang, R. de Callafon, M. Alma, Robust estimation and automatic controller tuning in vibration control of time varying harmonic disturbances, in: Proceedings of the 18th IFAC World Congress, Milano, Italy, 2011, pp. 5401-5406.

[8] B.Vau, I. Landau, Youla-kucera adaptive feedback disturbance rejection in the presence of plant uncertainties, in: Proceedings of the 58th IEEE Conf. on Decision and Control, Nice, France, 2019, pp. 102 107.

[9] S. Valentinotti, Adaptive rejection of unstable disturbances: Application to a fed-batch fermentation, Phd thesis, École Polytechnique Fédérale de Lausanne (April 2001).

[10] B.Vau, I. Landau, Adaptive rejection of narrow-band disturbances in the presence of plant uncertainties - a dual youla-kucera approach, Automatica To appear (available from https://hal.archives-ouvertes.fr/hal02959449)

[11] I. Landau, G. Zito, Digital control systems - Design, identification and implementation, Springer, London, 2005.

[12] I. Landau, A. Constantinescu, D. Rey, Adaptive narrow band disturbance rejection applied to an active suspension - an internal model principle approach, Automatica 41 (4) (2005) 563-574.

[13] I. D. Landau, R. Lozano, M. M'Saad, A. Karimi, Adaptive control, 2nd Edition, Springer, London, 2011.

[14] R. Melendez, I. Landau, L. Dugard, G. Buche, Data driven design of tonal noise feedback cancellers, in: Proceedings of the 20th IFAC World Congress, Toulouse, France, 2017, pp. 916-921. 